\title{
A Produção Científica Brasileira sobre Autismo na Psicologia e na Educação
}

\author{
Nelzira Prestes da Silva Guedes ${ }^{1}$ \\ Instituto Federal de Educação, Ciência e Tecnologia do Acre \\ Iracema Neno Cecilio Tada \\ Universidade Federal de Rondônia
}

\begin{abstract}
RESUMO - O autismo, embora tenha recebido destaque na literatura científica atual, ainda demonstra lacunas a serem investigadas. O presente artigo refere-se a uma revisão da literatura científica brasileira sobre o autismo nos campos da Psicologia e da Educação, com o intuído de identificar o atual cenário da produção científica sobre esta temática. Para tanto, foi realizado um levantamento de artigos científicos publicados em periódicos brasileiros no período de 2007 a 2012 . Foram recuperados 156 artigos, cujos resumos foram analisados e categorizados. Identificou-se que, em todas as categorias, esteve presente a sistematização das características do autismo ea elaboração de intervenções em prol da diminuição dos sintomas.
\end{abstract}

Palavras-chave: autismo, revisão de literatura, psicologia, educação

\section{Brazilian Scientific Production in Psychology and Education on Autism}

\begin{abstract}
Although autism has received special attention in current scientific literature, there are still gaps that need to be investigated. This article refers to a review of the Brazilian literature on autism in the fields of Psychology and Education, aiming to identify the current scenario of scientific literature on this topic. To this end, a survey was conducted of scientific articles published in Brazilian journals from 2007 to 2012. The survey included 156 articles, whose abstracts were analyzed and categorized. In all categories, a systematization was identified of the characteristics of autism and the development of interventions for the reduction of symptoms.
\end{abstract}

Keywords: autism, literature review, psychology, education

O autismo é uma condição caracterizada pelo desenvolvimento acentuadamente anormal e prejudicado nas interações sociais, nas modalidades de comunicação e no comportamento (American Psychiatric Association [APA], 2013). Tais características variam na maneira como se manifestam e no grau de severidade, estando dificilmente presente da mesma maneira em mais de uma pessoa.

Desde sua descoberta pelo médico austríaco Leo Kanner em 1943, este transtorno ou condição mental tem sido motivo de inúmeras discussões e controvérsias em relação ao seu diagnóstico, causas e tratamentos adequados. Sabese, por exemplo, que sua origem é determinada por fatores multicausais (Schwartzman, 2011a), mas não há respostas suficientes que os determinem, especificamente. No cenário científico, existem, por um lado, hipóteses de causas psicoafetivas, em que o autismo decorreria de impasses na constituição subjetiva do sujeito (Campanário \& Pinto, 2011; Rabello, 2004) ou de perturbações profundas na relação da criança com o meio (Hall, Nicholson, \& Adilof, 2006; Volk, Lurmann, Penfold, Hertz-Picciotto, \& McConnell, 2013). Por outro lado, existem hipóteses de causas neurobiológicas ou geneticamente determinadas, as quais priorizam proposições em que o autismo seria ocasionado por condições genéticas (Mecca et al., 2011), anormalidades cerebrais (Bolton, Griffiths, \& Pickles, 2002), alterações neuronais (Kooten et al., 2008; Wang et al., 2009) ou translocações cromossômicas (Tarelho \& Assumpção, 2007).

1 Endereço para correspondência: Instituto Federal de Educação, Ciência e Tecnologia do Acre. Estrada da APADEQ, n. 1192, Ramal da Fazenda Modelo, Bairro Nova Olinda. Cruzeiro do Sul, Acre, AC, Brasil. CEP: 69.980-000.E-mail: nelzira.guedes@gmail.com.
Tais vertentes científicas, muitas contraditórias entre si, formam a complexa conjuntura na qual o autismo está inserido, contribuindo para que muitas questões continuem na obscuridade. Desta forma, tal como aponta Klin (2006), o autismo tem permanecido um conceito heterogêneo que inclui múltiplos sintomas e uma variedade de manifestações clínicas em uma amplitude de níveis de desenvolvimento e de funcionamento, ainda que tenha havido recente tentativa de delimitá-lo e de simplificá-lo, como feito pela APA (2013).

No que tange à educação, as práticas atualmente reconhecidas às pessoas com autismo atuam sob um enfoque comportamental, estruturado a partir dos sintomas desta condição (Bragin, 2011; Orrú, 2012). No entanto, quando considerada a partir de um enfoque histórico-cultural, a educação se torna um processo de aprendizagem que impulsiona o desenvolvimento humano (Vygotski, 2000). Neste sentido, de acordo com Saviani (2011), a educação escolar tem o papel de transmissão do conhecimento cultural da humanidade de forma sistemática, conceituando-o formalmente. Em relação à pessoa com deficiência incluindo-se o autista -, por meio de situações dialógicas com significado cultural, e não com o foco na deficiência, é possível que o aluno com deficiência se aproprie do saber escolar e se humanize (Vygotski, 1997).

Diante das conjecturas apresentadas, acrescidas do recente e significativo crescimento do número de casos de autismo no mundo (Organização das Nações Unidas [ONU], 2003, 2010; Center for Disease Control and Prevention [CDC], 2013), verifica-se que estudos neste campo são necessários. 
O presente trabalho objetiva analisar a produção científica brasileira que aborda o tema autismo, a fim de que se identifique o atual cenário científico sobre esta temática nos campos da Psicologia e Educação. Para tanto, foram analisadas publicações no formato de artigos científicos publicados no período de 2007 a 2012.

\section{Método}

Foi realizado um levantamento bibliográfico nas bases eletrônicas de dados $S c i E L O$, PePSIC e Edubase, nas quais foram utilizadas como descritores de busca as palavraschave: autismo, transtornos globais do desenvolvimento, transtornos invasivos do desenvolvimento, transtornos do espectro do autismo, transtorno autista, e espectro autístico.

Aos critérios de inclusão foram considerados, além do ano de publicação, os artigos de periódicos brasileiros classificados como Qualis A e B nas áreas de Psicologia e Educação de acordo com a tabela de classificação QualisPeriódicos de 2012. Esta classificação refere-se a um conjunto de procedimentos utilizados pela Coordenação de Aperfeiçoamento de Pessoal de Nível Superior (CAPES) para avaliar os veículos da produção intelectual dos programas de pós-graduação em estratos indicativos de qualidade: $\mathrm{A} 1$, o mais elevado; A2; B1; B2; B3; B4 e; C, com peso zero.

Foram considerados artigos que apresentaram o tema autismo explícito no título ou no assunto do trabalho. Não foram incluídos trabalhos que empregaram o termo autismo em seu sentido lato, como sintoma de uma patologia, e tampouco resenhas de livros.

Os artigos selecionados tiveram seus resumos analisados e classificados em sete categorias: 1) Trabalhos de revisão e atualização teórica e sistemática; 2) Avaliação e caracterização das manifestações clínicas; 3) Estudos sobre prevalência e comorbidade; 4) Propriedades psicométricas de instrumentos de avaliação; 5) Autismo e relações familiares; 6) Intervenções e; 7) Escolarização e processos de ensino-aprendizagem. Apesar de alguns trabalhos estarem relacionados a mais de uma categoria, foram classificados apenas naquela a qual contempla o seu tema central.

\section{Resultados}

Foram identificados 156 artigos, distribuídos em 51 periódicos. A relação de publicações por ano segue a seguinte trajetória: em 2007 foram publicados 20 artigos; em 2008, 27 artigos; em 2009 e 2010, 35 e 28, respectivamente; em 2011, 27 artigos; enquanto no ano de 2012 foram publicados 19 artigos.

Pode-se perceber, conforme exposto na Figura 1, uma queda na produção científica nos últimos três anos em comparação com o período anterior, que vinha em um ritmo de crescimento.

Um aspecto identificado corresponde à faixa etária ou ao estágio de desenvolvimento dos estudos analisados. Dos 156 artigos identificados neste estudo, 96 declararam a faixa etária dos sujeitos estudados. Dentre eles, considerando a idade média nos estudos randomizados e amostrais, 79

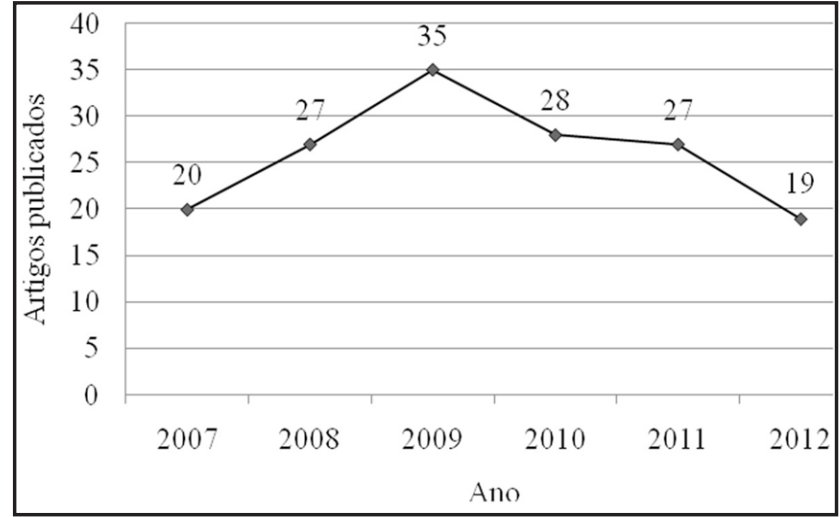

Figura 1. Distribuição dos artigos por data de publicação

abordavam a infância, 14 a adolescência e 3 a fase adulta, demonstrando o foco dos estudos brasileiros na primeira etapa de desenvolvimento. Considerou-se, para este estudo, o critério cronológico de desenvolvimento humano da legislação brasileira, na qual a infância é o período que contempla desde o nascimento aos doze anos incompletos, a adolescência dos 12 aos 18 anos, e a fase adulta da maioridade em diante (Brasil, 1988, 1990).

Os resumos dos artigos foram analisados e classificados em categorias de linha de pesquisa, as quais serão apresentadas a seguir. A Tabela 1 apresenta, de forma condensada, as características dos artigos selecionados, exibindo cada aspecto identificado nesta revisão, na qual é possível visualizar o quantitativo de artigos em cada temática.

\section{Trabalhos de sistematização, revisão e atualização teórica}

Nesta categoria foram incluídos 22 artigos, representando $14,1 \%$ do número total de trabalhos analisados. São estudos que discutem os aspectos gerais do autismo, seus critérios diagnósticos e abordam reflexões teóricas e críticas. São voltados, principalmente, para a discussão acerca da construção histórica e dos atuais paradigmas em relação ao autismo.

Nesta categoria, também foram incluídos os trabalhos que buscam a determinação dos possíveis fatores causais do autismo, os quais são relacionados a aspectos cognitivos, psicodinâmicos, orgânicos e genéticos. Com destaque em relação às proporções estatísticas, se encontram os trabalhos com enfoque na leitura psicanalítica da clínica do autismo, tema central de artigos dentro os identificados nesta categoria. São trabalhos que abordam essa condição como um sintoma subjetivo do sujeito.

\section{Avaliação e caracterização das manifestações clínicas}

O diagnóstico do autismo é difícil e complexo, feito por meio de investigação e da observação clínica do comportamento (Assumpção \& Kuczynski, 2011). Os estudos sobre a caracterização das manifestações comportamentais do autismo buscam definir seu perfil clínico e, assim, facilitar seu diagnóstico. Estão presentes, nesta categoria, 28 artigos 
Tabela 1. Descrição da Produção Cientifica sobre Autismo entre 2007 e 2012

\begin{tabular}{|c|c|c|c|}
\hline \multicolumn{2}{|c|}{ Características analisadas } & \multirow{2}{*}{$\begin{array}{c}\text { Número de } \\
\text { artigos }\end{array}$} & \multirow{2}{*}{ Total } \\
\hline Revisão e atualização teórica & Aspectos gerais (revisão bibliográfica) & & \\
\hline & Etiologia & 7 & 22 \\
\hline & Leitura psicanalítica & 8 & \\
\hline \multirow[t]{5}{*}{ Avaliação e caracterização das manifestações clínicas } & Perfil comportamental & 1 & \\
\hline & Perfil cognitivo & 7 & \\
\hline & Perfil sensório-motor & 11 & 28 \\
\hline & Perfil de comunicação & 6 & \\
\hline & Perfil de interação social & 3 & \\
\hline Prevalência e comorbidade & & & 5 \\
\hline Propriedades psicométricas de instrumentos de avaliação & & & 8 \\
\hline \multirow[t]{3}{*}{ Relações familiares } & Qualidade de vida do cuidador & 9 & \\
\hline & Impacto na dinâmica familiar & 5 & 18 \\
\hline & Relação mãe-filho & 4 & \\
\hline \multirow[t]{6}{*}{ Intervenções } & Tratamento farmacológico & 3 & \multirow{6}{*}{54} \\
\hline & Contexto institucional & 3 & \\
\hline & Intervenção precoce & 6 & \\
\hline & Intervenção psicopedagógica & 2 & \\
\hline & Intervenção psicoterapêutica & 16 & \\
\hline & Intervenção fonoaudiológica & 25 & \\
\hline \multirow[t]{4}{*}{ Escolarização e processos de ensino-aprendizagem } & Práticas educativas & 7 & \\
\hline & Ensino das habilidades acadêmicas & 2 & 21 \\
\hline & & 12 & \\
\hline & & & 156 \\
\hline
\end{tabular}

(17,9\% do número total de artigos analisados) voltados para a caracterização dos perfis comportamentais, cognitivos, sensório-motor, de comunicação e de interação social.

Dentre eles, um artigo abordou o perfil comportamental de crianças autistas, em uma tentativa de identificar problemas de comportamento. Os artigos que traçavam um perfil cognitivo do autista, o faziam em relação à memória, à formação de conceitos, à atribuição de estados mentais, à atenção e à compreensão idiomática.

Os estudos encontrados sobre perfis sensório-motor tinham foco, de modo mais específico: na caracterização dos potenciais evocados auditivos, a fim de identificar comprometimento da via auditiva em pessoas com autismo; no reconhecimento olfativo, buscando possíveis alterações na identificação de odores; nas habilidades viso-perceptuais e; na caracterização motora.

Os que tratavam dos perfis comunicativos buscavam classificar os aspectos da linguagem em crianças autistas, descrevendo sua funcionalidade e evolução, seu desempenho pragmático e o desenvolvimento da habilidade comunicativa em diferentes contextos situacionais. Já os artigos que caracterizavam o perfil de interação social, o faziam sob a tentativa de analisar a convivência dos autistas nas relações interpessoais, com foco no ambiente familiar, no desenvolvimento de brincadeiras com outras crianças e na habilidade de atenção compartilhada.

\section{Estudos sobre prevalência e comorbidade}

O autismo pode estar associado a diversas condições clínicas, tais como deficiência intelectual (presente em cerca de $50 \%$ dos casos de autismo), epilepsia (presente em até $42 \%$ dos casos), deficiência auditiva, síndrome de Down, dentre outras condições patológicas (Schwartzman, 2011b).

Em relação à prevalência, de acordo com os critérios de inclusão, o número pode variar de 2 a 113 casos de autismo a cada 10.000 habitantes (ONU, 2003, 2010), podendo alcançar números que chegam a uma proporção de um caso a cada grupo de 50 crianças, como apontado por uma pesquisa desenvolvida nos Estados Unidos pelo CDC (2013). No Brasil, poucos são os estudos que abordam a epidemiologia desta condição. Ribeiro (2007) realizou o primeiro estudo de prevalência de autismo na América Latina, identificando uma prevalência de $0,88 \%$ de casos de autismo em uma população de 1.470 crianças. O estudo de Ferreira (2008) estimou a frequência de autismo no estado de Santa Catarina, a partir das bases de dados de instituições especiais: Associações de Pais e Amigos dos Excepcionais (APAE), Associação de Amigos dos Autistas (AMA) e da Fundação Catarinense de Educação Especial (FCEE), nas quais foram identificadas uma prevalência de 1,31 de autistas a cada 10.000 pessoas. As limitações das pesquisas brasileiras demonstram a carência de dados epidemiológicos desta condição, que é considerada 
por alguns estudiosos como uma das mais frequentes desordens do desenvolvimento (Gadia, Tuchman, \& Rotta, 2004; The Autism Speaks, 2012; Brentani et al., 2013). Dados sobre esta questão contribuiriam para a elaboração de políticas que contemplem essa população.

Dentre os artigos analisados, cinco (3,2\% do número total) focaram na questão da prevalência e da comorbidade. Neste grupo estão os trabalhos sobre uma possível prevalência de doença celíaca no autismo e entre irmãos, sobre as associações entre o autismo e a epilepsia, bem como sobre sintomas do transtorno de déficit de atenção com hiperatividade em pacientes autistas.

\section{Propriedades psicométricas de instrumentos de avaliação}

O diagnóstico do autismo é emitido basicamente pelos critérios clínicos descritos na décima edição da Classificação Estatística Internacional de Doenças e Problemas Relacionados à Saúde (CID-10) e na quinta edição do Manual Estatístico e Diagnóstico da Associação Americana de Psiquiatria (DSM-V). Não existem exames ou procedimentos que confirmem isoladamente o seu diagnóstico, mas alguns que possibilitam a exclusão de outros quadros patológicos e a compilação de um número suficiente de informações que permitam esboçar mais seguramente o quadro clínico, tal como indicam Assumpção e Kuczynski (2011).

A utilização de instrumentos padronizados para fins de avaliação do autismo tem sido considerada uma necessidade como recurso alternativo que complemente as informações previamente adquiridas (Gadia et al., 2004; Léon \& Osório, 2011). Dentre eles destacam-se diversos questionários, checklists (listas de observação de comportamento), inventários e escalas especialmente desenvolvido para esta finalidade.

Oito artigos dentre os pesquisados $(5,1 \%$ do total analisado) abordam as propriedades psicométricas de instrumentos de avaliação. São estudos voltados para a reprodutibilidade de testes que possam auxiliar o diagnóstico concluso e precoce, referindo-se, essencialmente, à tradução, adaptação e validação de instrumentos de avaliação internacionais com intuído de torná-los aplicáveis à população brasileira. Os estudos englobam diversos testes, dentre os quais citam-se o Autism Diagnostic Interview - Revised (ADI-R), o Modified Checklist for Autism in Toddlers (M-CHAT), Childhood Autism Rating Scale (CARS) e o Autism Screening Questionnaire.

\section{Autismo e relações familiares}

Durante muito tempo, as causas do autismo foram delegadas às mães, uma vez que Kanner (1943) identificou como traço comum nas crianças por ele estudada a presença de mães frias e distantes. Apesar de o teórico, posteriormente, retratar-se por esta afirmativa, o conceito de autismo como um distúrbio originado pelo distanciamento afetivo da mãe já estava amplamente disseminado, chegando ao ponto de autores evidenciarem o autismo como decorrente de uma carência afetiva advinda de "mães-geladeira" (Bettelheim, 1987).

A família é considerada, atualmente, um suporte essencial para o desenvolvimento da pessoa com autismo junto às terapias multiprofissionais (Cunha, 2011; Silva, 2012).

Agruparam-se, nesta categoria, 18 estudos (11,5\% do total de artigos analisados), os quais discorrem sobre a qualidade de vida, níveis de estresse e de enfrentamento dos cuidadores de crianças com autismo, especialmente em relação às mães, sobre as mudanças, as dificuldades e o impacto na dinâmica familiar,e sobre a interação entre o autista e sua mãe.

\section{Intervenções}

O autismo é uma condição que é definida pela literatura como permanente e persistente, ou seja, não há uma cura e seus sintomas dificilmente são revertidos (Gadia et al., 2004; Klin, 2006; Schwartzman, 2011a, 2011b). De acordo com a Organização Mundial de Saúde [OMS] (2013), os sintomas do autismo podem dificultar seriamente o cotidiano das pessoas nessas condições e impedir realizações educacionais e sociais.

O que circula nestas literaturas é a concepção de que as condutas sociais dos autistas se desenvolvem de maneira inadequada para a sua cultura, principalmente no que diz respeito aos comportamentos de interação, devido à dificuldade de estabelecê-la ou mesmo à sua ausência. Tal conjuntura agrava-se pelos déficits de comunicação, também característicos desta condição (APA, 2013).

Não existe um tratamento farmacológico específico para o autismo, mas para as condições associadas a ele. Com relação às intervenções terapêuticas são indicadas as multiprofissionais, incluindo acompanhamentos psicológicos, fisioterápicos, fonoaudiológicos, neurológicos, psiquiátricos, nutricionais, além de inúmeros métodos e técnicas terapêuticas que podem auxiliar no tratamento do autismo (Silva \& Mulick, 2006; Schwartzman, 2011b). Desta forma, é possível alcançar a diminuição significativa dos sintomas, porém, os benefícios de cada tratamento específicos ainda são inconclusivos, com resultados, muitas vezes, limitados (Schwartzman, 2011c).

Dada a necessidade de intervenções a longo prazo, o autismo e as condições a ele associadas têm recebido atenção de políticas sociais, trazendo à tona questões de identificação e estimulação precoce, da natureza e intensidade dos tratamentos (Klin, 2006; Visani \& Rabello, 2012).

Foram incluídos nesta categoria 54 artigos (34,6\% do total analisado). Os estudos de intervenção aqui tratados são tanto estudos de caso quanto randomizados, com avaliação pré e/ ou pós-intervenção com intuito de comprovar a sua eficácia.

Os objetivos mais comuns encontrados foram: intervenções voltadas para a avaliação da eficácia de tratamento farmacológico, em casos de sintomas agressivos, de agitação psicomotora e de danos cardiovasculares; intervenções no contexto institucional, com propostas de tratamento a crianças e adolescentes institucionalizados; propostas, limites e possibilidades da intervenção precoce; intervenção psicopedagógica a partir da mediação lúdica 
por meio de vínculo interpessoal; bem como intervenções psicoterapêuticas, nas abordagens cognitivo-comportamental e psicanalítica.

Com número estatisticamente predominante, 25 artigos explanam sobre intervenções voltadas para o aprimoramento das funções comunicativas e avaliação de métodos terapêuticos fonoaudiológicos.

\section{Escolarização e processos de ensino-aprendizagem}

No Brasil, de acordo com Bragin (2011), a educação das pessoas com autismo, assim como das demais deficiências, foi oferecido inicialmente na educação especial, por meio de instituições especializadas. A partir das transformações nas políticas educacionais (Brasil, 2001, 2008), atualmente, a educação das pessoas com deficiência - abrangendo, neste sentido, os autistas -, tem sido priorizada a partir da perspectiva inclusiva.

Foram identificados 21 artigos (13,4\% do total analisado) com foco na temática da escolarização e dos processos educacionais, voltados, mais especificamente, para as práticas educativas, para o ensino de habilidades acadêmicas e para a inclusão escolar.

Os que tratam sobre as práticas educativas, o fazem sob enfoques diversos, abrangendo temas que se referem à relação entre os aspectos psiquiátricos do autismo como fator resultante de dificuldades escolares, à aplicação do programa TEACCH (Treatment and Education of Autistic and Related Communication Handicapped Children) na educação de alunos autistas, bem como à crítica a esse modelo e à defesa do ambiente digital de aprendizagem como facilitador no desenvolvimento da interação social de alunos com autismo.

Em relação aos trabalhos sobre o ensino das habilidades acadêmicas, foram identificados aqueles com enfoque no ensino da adição e da subtração a crianças com autismo e no ensino da leitura e da escrita como fim para a organização cognitiva, numa perspectiva psicanalítica.

Em relação à inclusão escolar e práticas inclusivas, foram identificados 12 artigos que discutem essa temática com o intuito de identificar os sentidos da inclusão escolar para professores e alunos e de relatar casos de práticas inclusivas.

\section{Discussão}

Os estudos analisados indicam a predominância da tentativa de sistematização das características e causas do autismo, divergindo entre si em diversos aspectos teóricos. Desta forma, pode-se considerar a dialética na qual o autismo está imerso, o que indica, por um lado, os avanços das pesquisas sobre a temática e, por outro, o quanto este tema ainda deve ser estudado. A ausência de respostas contundentes em relação aos fatores causais, bem como a amplitude sintomatológica que o autismo apresenta, se reflete nas tentativas de se sistematizar as manifestações clínicas do autismo.

Nesta perspectiva, de acordo com Schwartzman (2011a), as inúmeras causas identificadas para o autismo e as divergências neste campo de estudo demonstram a necessidade de que se entenda o autismo como uma condição multifatorial e de desenvolvimento de procedimentos que busquem a máxima redução dos prejuízos causados por esta condição.

Os estudos sobre intervenção são voltados, em sua maioria, às psicoterápicas e fonoaudiológicas. As intervenções psicoterápicas possuem uma perspectiva predominante na clínica psicanalítica, com foco na compreensão do autismo como oriundo da condição subjetiva do sujeito, constituído especialmente das relações mãe-bebê. As intervenções fonoaudiológicas focam no desenvolvimento da comunicação dos autistas, demonstrando, por um lado, a importância da linguagem nas relações sociais e, por outro, as dificuldades de desenvolvimento de novas formas de comunicação.

Neste sentido, vale considerar que estas linhas de pesquisas tendem a conceber o autismo sob uma única perspectiva, pautada nos sintomas decorrentes desta condição. Desconsideram a condição histórica do desenvolvimento humano e o contexto social como constituinte de suas características e de sua personalidade (Orrú, 2012; Vygotski, 2000).

Em relação à escolarização, foi observado que os estudos analisados atentam ao processo de inclusão escolar com vistas à interação social dos alunos autistas e à manipulação ambiental em prol de sua escolarização. Muitos são os estudos que desconsideram a escolarização como um processo de apropriação do saber científico, pressuposto defendido por Saviani (2011), embasando-se, essencialmente, nos sintomas do autismo, em especial, nas dificuldades de comunicação e de interação social dos autistas. Não são abordados modos alternativos de ensino, o que seria um ganho para o desenvolvimento de intervenções no meio escolar. Desta forma, constitui-se o que demonstra Orrú (2012), quando afirma que as práticas educativas voltadas para os autistas se estruturam no estigma de que, devido aos seus sintomas, nada, ou quase nada, pode ser feito por eles.

Outra questão a ser analisada é a prevalência de estudos que abordam o autismo sob o enfoque na infância. Estatisticamente, poucos são os artigos que se referem à adolescência e à fase adulta do autista. Estrutura-se, no campo científico, uma negligência a outras importantes fases do desenvolvimento humano, as quais carecem de concepções teóricas e de intervenções, concebendo um cenário em que "[...] curiosamente, a maioria das pessoas fala apenas de crianças autistas e nunca de adultos, como se de alguma maneira as crianças simplesmente sumissem da face do planeta" (Sacks, 2006, p. 248).

Esta conjuntura pode ser justificada pelas históricas concepções infantilizadoras da condição de deficiência que se refletem nas relações sociais e nas práticas escolares (Meletti, 2006; Vygotski, 1997).

\section{Considerações Finais}

A partir das múltiplas interpretações que impedem um consenso em relação ao desenvolvimento, constituição humana, intervenção e escolarização do autista, os dados presentes neste estudo demonstram a complexidade desta 
temática e as inúmeras possibilidades teóricas que dela decorrem.

O foco da produção científica brasileira sobre o autismo nos campos da Psicologia e da Educação tem se direcionado, essencialmente, para a identificação do autismo e para os modos de intervenção, priorizando percepções unilaterais de aspectos isolados do autismo, sem considerar a complexidade da constituição histórica e social em todas as fases do desenvolvimento humano.

Nesta perspectiva, são necessários estudos que foquem não apenas a sintomatologia do autismo e que somente por meio dela sejam propostas intervenções, mas estudos que, considerando as dificuldades e as potencialidades dessas pessoas, lancem propostas que possam, antes de tudo, promover uma verdadeira inclusão social dos autistas e, portanto, maior qualidade de vida para eles.

As divergências neste campo teórico não constituem um limite teórico-prático desta condição, mas, considerando sua condição dialética, pode representar uma possibilidade da existência de diferentes soluções para as diversas questões suscitadas no atendimento e acompanhamento de pessoas com autismo.

Considerando as limitações deste estudo, é necessário maior aprofundamento no cenário científico sobre o autismo, bem como solidificação da produção científica nacional neste campo.

\section{Referências}

American Psychiatric Association. (2013). DSM-5 Autism Spectrum Disorder Fact Sheet. American Psychiatric Publishing. Recuperado em 5 de dezembro de 2013, de http://www. dsm5.org/Documents/Autism\%20Spectrum\%20Disorder\%20 Fact\%20Sheet.pdf.

Assumpção. F., Jr., \& Kuczynski, E. (2011). Psicofarmacoterapia nos transtornos globais do desenvolvimento. In J. Schwartzman \& C. Araújo (Eds.), Transtornos do Espectro do Autismo (pp. 215-226). São Paulo: Memmon.

Bettelheim, B. (1987). A fortaleza vazia. São Paulo: Martins Fontes.

Bolton, P., Griffiths, P., \& Pickles, A. (2002). Neuro-epileptic determinants of autism spectrum disorders in tuberous sclerosis complex. Brain, 125(6), 1247-1255.

Bragin, J. (2011). Antecedente da educação de autistas no Brasil: teorias políticas e suas influências nas práticas pedagógicas em centros de atendimento educacional especializado. Revista Digital Fermentario, 5, 1-22. Retrievedfromhttp:// www.fermentario.fhuce.edu.uy/index.php/fermentario/article/ view/60/17.

Brasil (1988). Constituição da República Federativa do Brasil. Brasília, DF: Senado.

Brasil (1990). Estatuto da criança e do adolescente. Brasil: Presidência da República, 1990. Retrievedfromhttp://www. planalto.gov.br/ccivil_03/leis/18069.htm.

Brasil. (2001). Diretrizes nacionais para a educação especial na educação básica. MEC/SEESP. Retrievedfromhttp://portal. mec.gov.br/seesp/arquivos/pdf/diretrizes.pdf,
Brasil (2008). Politica nacional de Educação Especial na perspectiva da Educação Inclusiva.Brasília: MEC/SEESP. Retrievedfromhttp://peei.mec.gov.br/arquivos/politica_ nacional_educacao_especial.pdf.

Brentani, H., Paula, C., Bordini, D., Rolim, D., Sato., F., Portolese, J., Pacífico, M., \& McCracken, J. (2013). Autism spectrum disorders: an overview on diagnosis and treatment. Revista Brasileira de Psiquiatria, 35, 62-72.

Campário, I.,\& Pinto, J. (2011). Devastação e autismo. Estudos de Psicanálise, 36, 93-102.

Center for Disease Control and Prevention. (2013). Changes in prevalence of parent-reported autism spectrum disorder in school-aged U.S. children: 2007 to 2011-2012. National Health Statistics Reports, 65.

Cunha, E. (2011). Autismo e Inclusão: psicopedagogia e práticas educativas na escola e na família ( $3^{\mathrm{a}}$ ed.). Rio de Janeiro: Wak Ed.

Ferreira, E. (2008). Prevalência de autismo em Santa Catarina: uma visão epidemiológica contribuindo para a inclusão social. (Unpublished master'sthesis). Universidade Federal de Santa Catarina, Florianópolis, SC, Brasil.

Gadia, C., Tuchman R., \& Rotta, N. (2004). Autismo e doenças invasivas de desenvolvimento. Jornal de Pediatria, 80 (supl. 2), 83-94. Retrievedfromhttp://www.scielo.br/scielo. php?pid=S0021-75572004000300011\&script=sci_arttext.

Hall, M., Nicholson, S., \& Adilof, N. (2006). Does television cause autism? National Bureau of Economic Research Working Paper Series, 12632, 1-47.

Kanner, L. (1943). Autistic disturbance of affective contact. Nervous Child, 2, 217-250.

Klin, A. (2006). Autismo e síndrome de Asperger: uma visão geral. Revista Brasileira de Psiquiatria, 28(1), 3-11. Retrievedfromhttp://www.scielo.br/scielo.php?script=sci arttext\&pid $=$ S1516-44462006000500002\&lng $=$ en\&nrm $=\overline{\text { is }}$ o\&tlng=pt.

Kooten, I. van., Palmen, S. Cappeln, P. von., Steinbusch, H., Koor, H., Heinsen, H., Hof, P., Engeland, H. van \& Schmitz, C. (2008). Neurons in the fusiform gyrus are fewer and smaller in autism. Brain, 131(4), 987-99.

Leon, V. \& Osório, L. V. C. (2011). O método TEACCH. In J. Schwartzman \& C. Araújo (Eds.), Transtornos do Espectro do Autismo (pp. 263-277). São Paulo: Memmon.

Mecca, T., Bravo, R., Velloso, R., Schwartzman, J., Brunoni, D.,\& Teixeira, M. (2011). Rastreamento de sinais e sintomas de transtornos do espectro do autismo em irmãos. Revista de Psiquiatria do Rio Grande do Sul, 33(2), 116-120.

Meletti, S. (2006). Educação da pessoa com deficiência mental em instituições de educação especial: da política à instituição concreta. (Unpublished doctoral dissertation). Universidade de São Paulo, São Paulo, SP, Brasil.

Organização das Nações Unidas (2003). Weekly epidemiological Record, 78(4). Retrievedfromhttp://www.who.int/wer/2003/ en/wer7804.pdf.

Organização das Nações Unidas (2010, April 2). Greater awareness and understanding of autism needed, says UN chief. UN News Centre. Retrievedfromhttp://www.un.org/apps/news/story. asp?NewsID=34272\#.UgQq51c5fIU. 
Organização Mundial de Saúde. (2013). Autism spectrum disorders \& other developmental disorders: from raising awareness to building capacity. Meeting report, Geneva. Retrievedfromhttp://apps.who.int/iris/bitstre am/10665/103312/1/9789241506618_eng.pdf.

Orrú, S. (2012). Autismo, linguagem e educação: integração social no cotidiano escolar (3a ed.). Rio de Janeiro: WAK Ed.

Rabello, S. (2004). Sobre crianças e autistas. Textura Revista de Psicanálise, 4(4), 48-51.

Ribeiro, S. (2007). Prevalência dos transtornos invasivos do desenvolvimento no município de Atibaia: um estudo piloto. (Unpublished master'sthesis). Universidade Presbiteriana Mackenzie, São Paulo, SP, Brasil.

Sacks, O. (2006). Um antropólogo em Marte: sete histórias paradoxais (Bernardo Carvalho, Trad.). São Paulo: Companhia das Letras. (Trabalho original publicado em 1995)

Saviani, D. (2011). Pedagogia histórico-crítica: primeiras aproximações (11a ed. rev.). Campinas: Autores Associados.

Schwartzman, J. (2011a). Transtornos do espectro do autismo: conceitos e generalidades. In J. Schwartzman \& C. Araújo (Eds.),Transtornos do Espectro do Autismo (pp. 37-42). São Paulo: Memmon.

Schwartzman, J. (2011b). Condições associadas aos Transtornos do Espectro do Autismo. In J. Schwartzman \& C. Araújo (Eds.), Transtornos do Espectro do Autismo (pp. 123-143). São Paulo: Memmon.
Schwartzman, J. (2011c). Terapias alternativas e controversas. In J. Schwartzman \& C. Araújo (Eds.), Transtornos do Espectro do Autismo (pp. 255-262). São Paulo: Memmon.

Silva, A. (2012). Mundo singular: entenda o autismo. Rio de Janeiro: Editora Objetiva.

Silva, M., \& Mulick, J. (2006). Diagnosticando o transtorno autista: aspectos fundamentais e considerações práticas. Psicologia: Ciência e Profissão, 29(1), 116-131.

Tarelho, L., \& Assumpção, F., Jr. (2007). A case of pervasive devolopmental disorder with chromosomal translocation 1-4. Arquivos de Neuro-Psiquiatria, 65(1), 153-156.

The AutismSpeaks. (2012). What is autism? Retrievedfromhttp:// www.autismspeaks.org/what-autism.

Visani, P. \& Rabello, S. (2012). Considerações sobre o diagnóstico precoce na clínica do autismo e das psicoses infantis. Revista Latinoamericana de Psicopatologia Fundamental, 15(2), 293-308.

Volk, H., Lurmann, F., Penfold, B., Hertz-Picciotto, I. \& McConnell, R. (2013). Traffic-Related Air Pollution, Particulate Matter, and Autism. JAMA Psychiatry, 70(1), 71-77.

Vygotski, L. (1997). Obrasescogidas V: Fundamentos de Defectología. Madrid: Visor.

Vygotski, L. (2000). Obrasescogidas III: Problemas del desarrollo de la psique ( $2 \mathrm{a}$ ed.). Madrid: Visor.

Wang, K., Zhang, H., Ma, D., Bucan, M., Glessner, J., Abrahams, B., ... ...Hakonarson, H. (2009). Common genetic variants on $5 \mathrm{p} 14.1$ associate with autismspectrum disorders. Nature, 459(7246), 1-10. 